\title{
O desenvolvimento da Lógica Mental Predicativa entre crianças de diferentes níveis sócio-econômicos
}

\author{
Maria da Graça Bompastor Borges Dias \\ Avany Bernardino Corrêa Sobral \\ Lisandra Mendonça de Carvalho \\ Universidade Federal de Pernambuco
}

\begin{abstract}
Resumo
O modelo da lógica mental predicativa desenvolvido por Braine e O'Brien (1998) é uma extensão da teoria da lógica proposicional. A lógica predicativa inclui termos quantificadores tais como "todos", "a maioria", "alguns", "nenhum", entre outros. De acordo com Braine (1978), a lógica mental nãoé equivalente ao pensamento lógico formal na escola. A compreensão das crianças de quantificadores universais, portanto, fornece subsídios para a construção de hipóteses sobre a natureza da lógica mental. No presente estudo, crianças de 4 a 7 anos de idade, de níveis sócio-econômicos médio e baixo, estudantes de escolas particulares e públicas, foram entrevistadas utilizando-se para tal um conjunto com 4 desenhos, correspondentes aos 4 quantificadores. As crianças foram instruídas a indicar a figura relacionada à sentença lida. As análises estatísticas mostraram que o quantificador "nenhum" obteve o maior número de acertos, diferindo significativamente dos outros três. O quantificador "todos" obteve maior média de acertos do que "alguns" a "maioria". As crianças de 4 anos ofereceram menos acertos que as outras idades, como também ocorreu com aquelas de nível sócio-econômico baixo em relação às do nível sócio-econômico médio.
\end{abstract}

Palavras-chave: Lógica Mental Predicativa, Quantificadores, Proposições.

\begin{abstract}
The development of Predicate Mental Logic among children from public and private schools. The model of the Predicate Mental Logic developed by Braine and O'Brien (1998) is an extension of the Proposition Mental Logic. The predicative logic include quantifiers as "all", "the majority", "some", "none", among others. According to Braine (1978), the mental logic is not equivalent to the formal logic thaught in the school. Children's comprehension of universal quantifiers therefore, provides subsidy to buiding hypothesis about the mental logic nature. In this study, children from 4 to 7 -yearold, of medium and low socioeconomic levels, of private and public schools, were interviewed with a set with 4 drawings, corresponding to the 4 quantifiers. It was asked to the children to indicate the figure relating to the read sentence. The statistic analysis showed that the quantifier "none" obtained significant more correct responses than the others three. The quantifier "all" obtained more correct responses than "some" and "the majority". The 4-year-olds offered less correct responses than the other ages, the same occured with children from low socioeconomic level when compared to those from medium socio-economic level.
\end{abstract}

Key-Words: Predicative Mental Logic, Quantifiers, Propositions.

\section{A Teoria da Lógica Mental}

$\mathrm{E}$ xiste controvérsia sobre a extensão da capacidade de crianças e adultos compreenderem apropriadamente condicionais, isto é, proposições na forma "se p então q". De um lado encontra-se Evans (1982) que argumenta que "o desempenho indica não mais que uma com- preensão superficial da sentença 'se p então q ', e pouca evidência de qualquer compreensão mais profunda” (p. 231). No outro extremo encontramos vários pesquisadores que mostram evidências de que mesmo crianças bem novas compreendem a lógica de condicionais (e.g. Brainerd, 1977; Dias, 1996; Dias \& Harris, 1988, 1990; Dias \& Ruiz, 1990; Ennis, 
1971, 1975; O’Brien, Braine, Connell, Noveck, Fish \& Fun, 1989). Entre essas duas posições estão aqueles que advogam que adultos e adolescentes denotam compreensão adequada dos condicionais, enquanto as crianças apresentam a compreensão dos mesmos incompleta e insuficiente. A compreensão de condicionais por adultos seria então qualitativamente diferente da compreensão apresentada por crianças (e.g. Knifong, 1974; Kuhn, 1977; Markovits, 1984, 1985, 1993; Matalon, 1962; Moshman, 1979; Overton, 1990; Paris, 1973; Standenmayer \& Bourne, 1977; Taplin, Standenmayer \& Taddonio, 1974; Ward \& Overton, 1990).

Essa controvérsia, para O'Brien, Dias, Roazzi e Braine (1998), pode ter raízes nos diferentes tipos de tarefas de raciocínio utilizados. Os estudos concluem que tanto crianças como adultos não são capazes de uma compreensão apropriada dos condicionais. Os pesquisadores de tais estudos usaram a tabela da verdade, para verificar o desempenho dos sujeitos. Segundo O'Brien et al. (1998), essa tabela não captura o que os indivíduos entendem sobre condicionais.

\footnotetext{
... vendo os condicionais através das lentes da tabela de verdade obscurece o papel dos condicionais no raciocínio comum: se indica suposição e um raciocinador comum supõe algo para descobrir o que deveria ser o caso sob essa suposição. O raciocínio condicional assim preocupase com situações hipotéticas e de faz-de-conta que encontram-se subjacentes a ele. Enquanto o material condicional não captura esta natureza suposicional do raciocínio condicional, a visão de condicionais na perspectiva da lógica mental dos esquemas de inferências, assim o fazem (p. 246).
}

O esquema da lógica mental para a prova condicional juntamente com raciocínio direto rotineiro (ver mais adiante), fornecem um procedimento semântico para "se", isto é, um procedimento para julgar quando proposições condicionais são verdadeiras ou falsas. Quando um condicional está sendo avaliado, a rotina do raciocínio direto adiciona seu antecedente ao conjunto de premissas e trata o conseqüente como uma conclusão a ser avaliada. Assim, "se p então q" é julgado verdadeiro quando o conseqüente "q" é verdadeiro na premissa juntamente com a suposição de "p" (Modus Ponens). No entanto, "se p então q" é julgado falso quando o conseqüente "q" é falso na premissa com a suposição de "p". Na lógica padrão este último julgamento não deveria ser realizado por ser o antecedente falso. Também na lógica padrão pode-se derivar qualquer conclusão quando as premissas são falsas, isto é, qualquer conclusão segue-se de uma contradição. Já na lógica mental nada se segue nessa situação. E quando uma suposição leva à uma contradição, esta é julgada falsa.

A teoria da lógica mental proposicional desenvolvida nas últimas duas décadas (Braine \& O'Brien, 1991; Braine,
O’Brien, Noveck, Samuels, Lea, Fish \& Yang, 1995; O’Brien, 1993, 1995), fornece uma explicação do tipo de inferências dedutivas que os sujeitos fazem a partir de partículas lógicas como "se", "e", "não" e "ou”. A lógica proposicional consiste em um conjunto de esquemas de inferências lógicas que dizem respeito à conjunção, disjunção, negação e condicionais, e um programa de raciocínio para a aplicação dos esquemas. Os estudos realizados por esses pesquisadores averiguaram as inferências dos sujeitos durante processamento de textos, discursos, como também quando os mesmos resolvem problemas silogísticos. A maioria desses estudos procurou evidências desses esquemas lógicos proposicionais em adultos (e.g. Lea, 1998; Lea, O’Brien, Fish, Noveck \& Braine, 1990). Entre crianças, temos apoios ao modelo através dos trabalhos de Bloom, Lahey, Hood, Lifter e Feiss (1980); Bowerman (1986); Braine e Rumain (1981); O'Brien, Braine, Cornell, Noveck, Fish e Fun (1989); O'Brien, Dias, Roazzi e Braine (1998).

A teoria da lógica mental apresenta três partes que serão aqui discutidas brevemente. Uma parte consiste de um conjunto de esquemas de inferências como, por exemplo, quando alguém sabe que duas proposições na forma "p" ou "q" e "não p" são verdadeiros, "q" pode ser concluído. O conjunto de esquemas básicos que aqui importa é aquele que está incluído nas inferências lógicas que os indivíduos fazem rotineiramente e não todos os tipos que eles seriam capazes de fazer.

A segunda parte da teoria consiste em um programa de raciocínio que aplica os esquemas em linhas de raciocínio e inclui uma rotina direta (raciocínio mais simples) e uma estratégia mais sofisticada (raciocínio indireto). A primeira linha seria universal e aplicável com esforço mínimo tanto no raciocínio como na compreensão. Segundo os defensores dessa teoria, pode-se predizer que as inferências que são elaboradas, aplicando-se os esquemas básicos com o raciocínio direto, são feitas rotineiramente. Esses são aplicados corretamente mesmo por crianças pré-escolares (O’Brien, Dias, Roazzi \& Braine, 1998).

Esse tipo de esquema para prova condicional ${ }^{1}$ está avaliável logo cedo devido ao uso de suposição nas brincadeiras de faz-de-conta das crianças e também de seus usos espontâneos com "se". Evidências sobre o bom desempenho de crianças pré-escolares foram também demonstradas nos estudos de Dias e colaboradores (Dias, 1996; Dias \& Harris, 1988; 1990; Dias \& Ruiz, 1990). Nesses estudos, crianças de até 3 anos de idade foram capazes de fazer inferências corretamente do tipo Modus Ponens, mesmo com conteúdos de premissas incongruentes com a realidade diária, desde que os problemas fossem apresentados em um contexto fantasioso de brincadeira de faz-de-conta. Bowerman (1986), Fein (1981) e Reilly (1986), verificaram o uso freqüente de 
suposições espontâneas quando crianças de 3 anos brincavam. Leslie (1987) relata a utilização de inferências simples em brincadeira de faz-de-conta entre crianças com menos de 2 anos de idade.

O'Brien, Dias, Roazzi e Braine (1998) argumentam ainda, que existem evidências para estratégias mais sofisticadas entre universitários e, presumivelmente, em outras populações, porém essas não seriam universais e seriam menos freqüentes que as estratégias básicas. "As estratégias de raciocínio indireto são capacidades secundárias adquiridas mais tarde, que são sujeitas à variação individual embora comum em sujeitos adultos.” (Braine \& O'Brien, 1998, p. 79)

A terceira parte da teoria é pragmática. A teoria propõe que o significado lógico básico de cada partícula encontrase nas inferências que são sancionadas pelos esquemas de inferências. Os esquemas de inferências atuariam nas representações semânticas que seriam ooutput dos processos de compreensão. Logo, os fatores pragmáticos que influenciam a compreensão são afetados pela informação através da qual as inferências são realizadas.

Há então três princípios gerais:

1. A estratégia da plausibilidade da interpretação da sentença: os sujeitos interpretaram mais acuradamente uma proposição quando esta envolveu seus conhecimentos específicos e gerais do mundo.

2. Princípio da cooperatividade: o falante tenta ser verdadeiro o quanto possível, informativo, claro em suas exposições e, do mesmo modo, o ouvinte interpreta as exposições assumindo que as mesmas são verdadeiras, informativas etc. Por este motivo, esse princípio oferece margem a muitas inferências desnecessárias (implicações conversacionais), que podem ser uma fonte de erros em tarefas lógicas.

3. Provocação de inferências: por exemplo, um condicional "se p então q" provoca e a inferência "se não p então não q"; uma sentença com ou chamaria uma inferência com "ambos não", e assim por diante (conversão inválida). Os sujeitos fazem erros deste tipo a não ser que haja algum contramando que mostraria ser inapropriado (Dias, 1996). Em vários estudos, esta autora tenta minimizar este tipo de erro, tanto entre criança como entre adultos, utilizando uma sentença do mesmo grupo. Por exemplo, Dias e Ruiz (1990) em estudo com crianças entre 5 a 8 anos, expandiram a premissa maior com uma frase exemplificando outro fato pertencente a uma mesma categoria, como no seguinte silogismo envolvendo a afirmação do conseqüente (forma inválida).

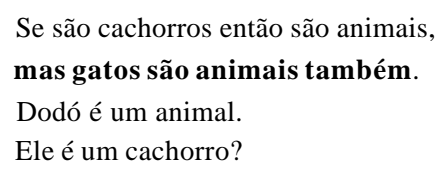

Nesse estudo, houve uma melhora significativa no desempenho das crianças, tanto nas formas válidas (Modus
Ponens e Modus Tollens), como nas inválidas (Afirmação do Conseqüente e Negação do Antecedente). Nas formas inválidas os resultados foram sempre mais baixos do que nas válidas. Resultados similares foram alcançados por Rumain, Cornell e Braine (1983) quando a premissa maior era expandida (complexa), como por exemplo:

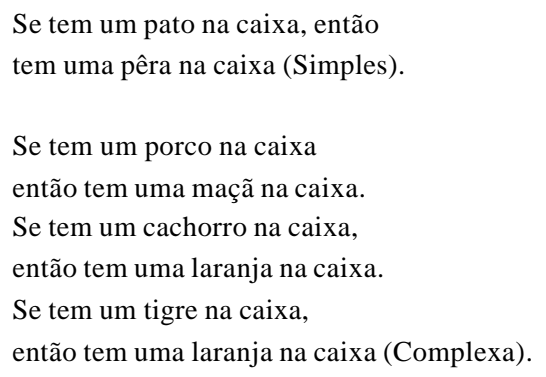

Já a Teoria da Lógica Mental Predicativa desenvolvida recentemente (Braine \& O'Brien, 1998), e sendo uma extensão da teoria da lógica mental proposicional, inclui termos quantificadores como "todos", "alguns", "nenhum", "a maioria", "poucos" etc.

O modelo da lógica predicativa também fornece instrumentos para analisar a estrutura interna das proposições (em termos da estrutura predicado/argumento) e para a quantificação (usando uma estrutura pronominal do tipo encontrado nas linguagens naturais no lugar da perspectiva visada pelos sistemas da lógica padrão oustandard).

A teoria propõe que o estímulo da linguagem natural é traduzido na linguagem da lógica mental. A lógica mental inclui um conjunto de esquemas inferenciais que fornecem conclusões para serem inferidas a partir da informação pressuposta como verdadeira. Por exemplo, a informação que "os meninos ou brincaram com as meninas ou eles brigaram com as meninas" será transformado como $\mathrm{S} 1$ [Todos X]OU $\mathrm{S} 2$ [PRO-Todos X], onde S1= "brincaram com as meninas", $\mathrm{S} 2=$ "brigaram com as meninas", e X= "meninos". Aplicações de um destes esquemas da teoria leva a $\mathrm{S} 2[$ Todos X: NEG S1[PRO]], que pode ser traduzido de volta para o português como, "os meninos que não brincam comasmeninas brigaram com elas". A teoria faz predições precisas sobre (a) qual problema será resolvido, (b) os passos exatos nos quais inferências intermediárias são feitas no processo de solução destes problemas, e (c) a relativa dificuldade com a qual os problemas serão percebidos.

A compreensão que as crianças têm dos quantificadores é um domínio ideal para a construção de hipóteses sobre a natureza da lógica mental. Isto, em virtude dos quantificadores fornecerem a base para um conjunto de inferências lógicas. Uma maneira para discernir a estrutura da lógica mental é investigar as representações semânticas que servem de base para expressões naturais da língua, inclusive os quantificadores. 
Os quantificadores estão presentes em muitas línguas com funções semânticas análogas aos quantificadores da língua inglesa "all", "none", "the majority", "each", "some" etc. (Ioup, 1975)

A interpretação de "todos", por exemplo, para a sentença "todas as bolas estão na caixa", é que todas as bolas estão dentro da mesma caixa e nenhuma está fora. A sentença "algumas bolas estão na caixa", leva à interpretação que umas estão na caixa e outras não. Já para "a maioria das bolas estão na caixa”, resulta na interpretação que só algumas não estão na caixa e mais bolas estão dentro da caixa. Quanto a "nenhuma bola está na caixa" deve ser interpretada que todas as bolas estão fora da caixa e a caixa está sem bola.

Para Gelman e Meck (1983), essas distinções semânticas seriam controladas desde cedo no desenvolvimento das crianças. Isto aconteceria através de experiências rotineiras tais como, atividades de contar que fazem correspondência um a um e organizar objetos em grupos.

Braine (1978) enfatizou que a lógica mental não é equivalente ao pensamento lógico formal na escola e a compreensão das crianças de quantificadores universais fornece subsídios para a construção de hipóteses sobre a natureza da lógica mental. Com esse propósito em mente, alguns estudos foram desenvolvidos. O estudo de Brooks, Braine, Jia e Dias (1998) procurou comparar a aquisição do vocabulário associado à quantificação universal entre crianças e adultos falantes nativos do inglês, chinês e português. Com isto, os autores procuraram restringir hipóteses dos tipos de representações associados ao vocabulário lógico (ver também Brooks, Braine, Jia \& Dias, 2001).

Também o estudo de Dias e Brooks (1997) procurou descrever o desenvolvimento de mapeamento entre quantificadores universais "todos" e "cada" e a interpretação de sentenças em crianças falantes da língua portuguesa. Os dados sugerem que noções com

\begin{abstract}
significado coletivo ou de "grupo" associado com todos e seus correspondentes em outras línguas, e o significado distributivo ou em "pares" associado com cada e seus correspondentes, são prováveis candidatos primitivos na sintaxe do pensamento subjacente a uma lógica preditiva natural. Pode-se ter em mente que as crianças não estão simplesmente ganhando competência em associarem pistas lingüísticas com interpretações distributivas e coletivas, mas estão elaborando um sistema inteiro de raciocínio dedutivo (p.110).
\end{abstract}

Como já foi relatado anteriormente, o modelo da lógica mental predicativa estende a teoria da lógica proposicional incluindo termos quantificadores tais como "todos", "alguns", "nada", "nenhum", "a maioria", "poucos", entre outros.

Perguntamos, então: será que esses quantificadores seriam adquiridos pelas crianças em uma mesma idade ou ha- veria uma ordem de aquisição? Será que a rotina direta seria primeiramente empregada a alguns desses quantificadores e, posteriormente, em outros? Será também interessante verificar se existe diferença de desempenho entre diferentes níveis sócio-econômicos que estão diferenciados em creches e escolas públicas e particulares aqui escolhidas.

Assim, este estudo é uma investigação do desenvolvimento da compreensão dos quantificadores, tendo como objetivo descrever o desenvolvimento da compreensão dos mesmos e a interpretação de sentenças em crianças de diferentes níveis sócio-econômicos.

\section{Método}

\section{Participantes}

Fizeram parte do estudo 80 crianças de 4 a 7 anos de idade, sendo 20 de cada idade. As crianças foram selecionadas em escolas particulares que atendem ao nível sócio-econômico médio (40) e igual número em creches e escolas públicas que atendem ao nível sócio-econômico baixo. O critério utilizado para definição do nível sócio-econômico (NSE) dos participantes foi estabelecido a partir do tipo de escola que a criança frequientava, podendo ser pública (NSE baixo) ou particular (NSE médio).

\section{Material e Procedimento}

Treinamento: um conjunto de quatro desenhos (Apêndice).

Tarefa:

Desenhos:

Foi utilizado um conjunto com quatro desenhos, sendo que um dos desenhos apresentava o quantificador "todos", outro, o quantificador "alguns", o terceiro, "a maioria" e o quarto, "nenhum" (ver Figura 1).

Sentenças:

Quatro tipos de sentenças (ver também Figura 1) foram apresentados, com um único quarteto de desenhos.

Para o treinamento foi utilizado material similar ao acima exposto. As crianças foram entrevistadas individualmente. Primeiramente foram mostrados a cada criança quatro desenhos e pedia-se: "Aponte um só desenho que mostra que todas as crianças estão dentro da piscina".

Em alguns casos, a criança apontava os quatro desenhos. Nestas ocorrências o examinador explicava que apenas um desenho mostrava o que havia sido pedido. Caso a criança falhasse novamente, o examinador explicava cada um dos desenhos, por exemplo: "Preste atenção neste desenho. Tem duas criancinhas dentro e duas criancinhas fora. Você pode dizer que todas as crianças estão dentro da piscina?". Vale ressaltar que em apenas cinco casos (todos entre crianças de 4 anos) essas explicações foram necessárias. 
1 - Todas as meninas estão alimentando um cachorrinho

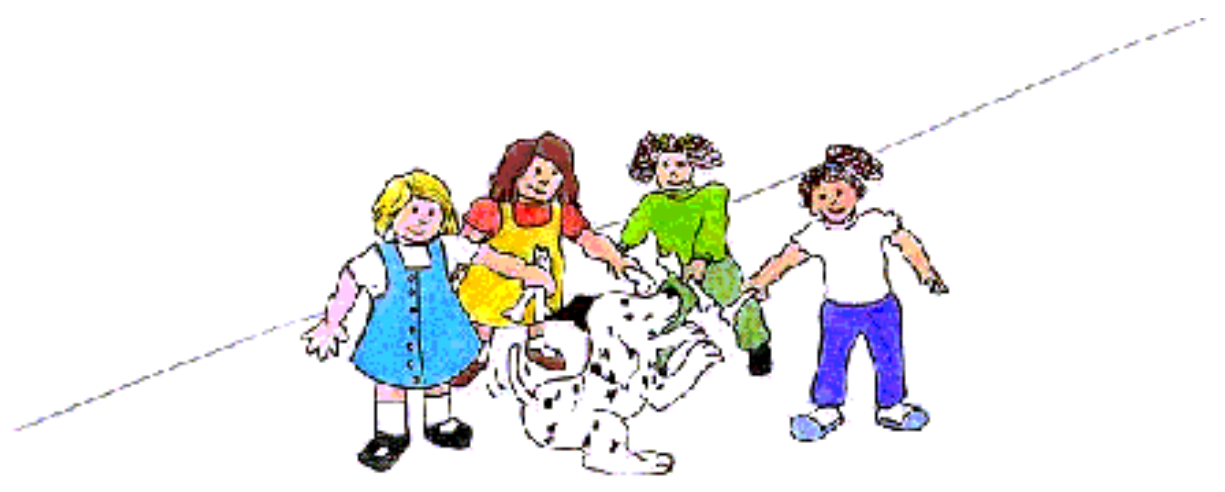

2 - A maioria das meninas está alimentando um cachorrinho

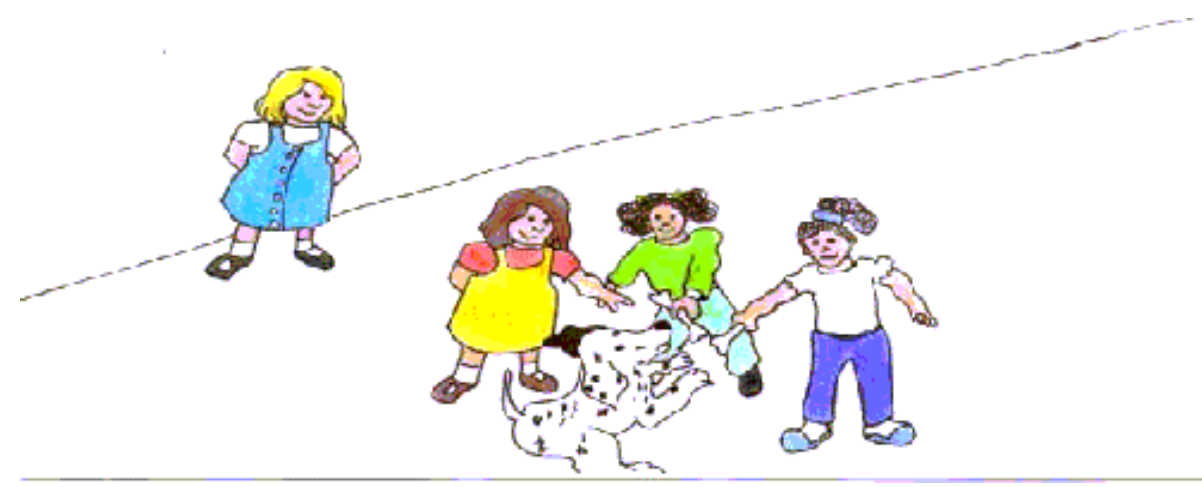

3 - Algumas meninas estão alimentando um cachorrinho

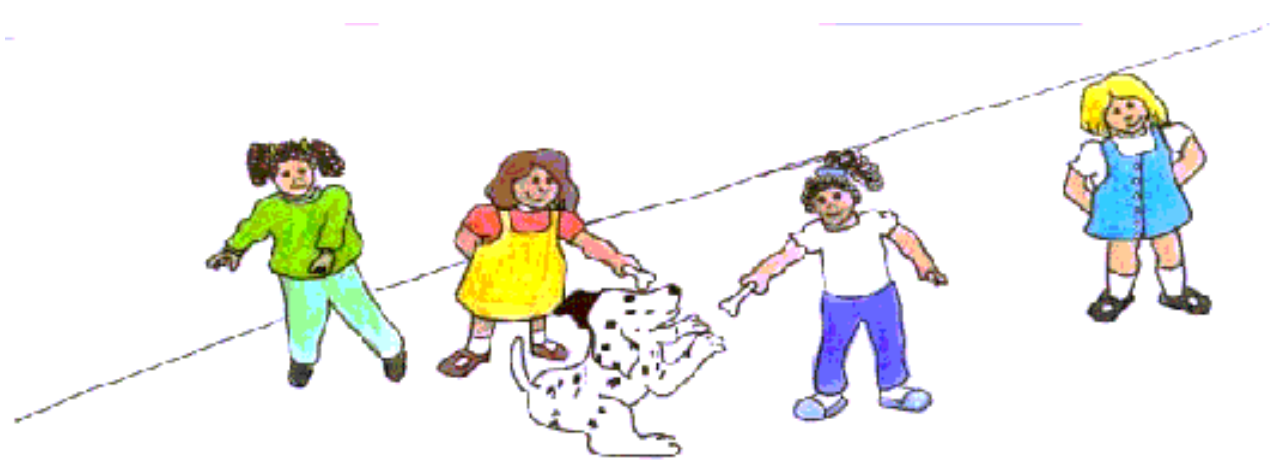

4 - Nenhuma menina está alimentando um cachorrinho

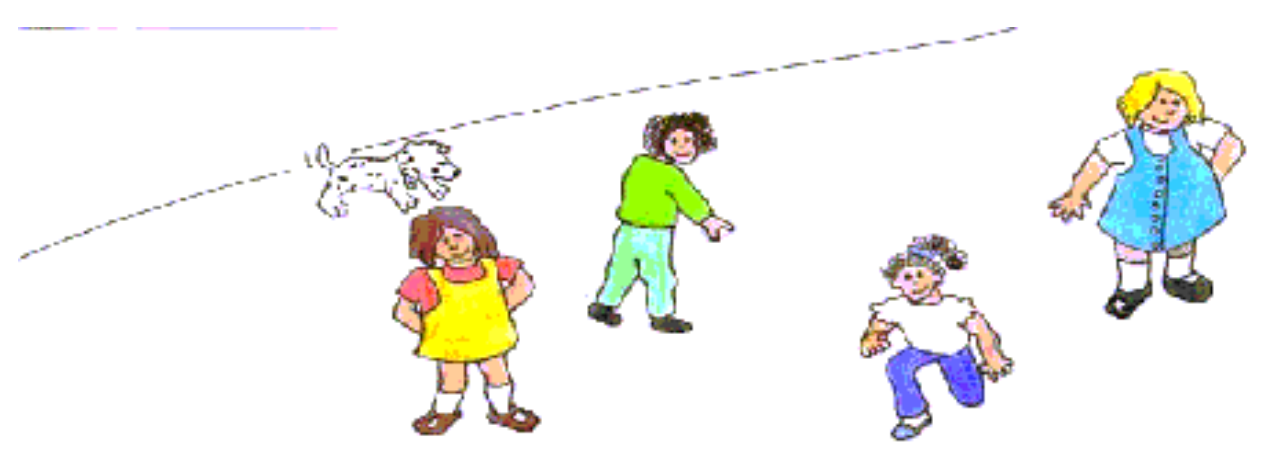


Quando a criança acertava, era apresentada a tarefa propriamente dita.

Este treinamento foi utilizado para certificar-se de que as crianças compreendiam o procedimento da tarefa.

Para a tarefa, o examinador apresentava os quatros desenhos de uma só vez e pedia à criança para escolher a figura que melhor representava a sentença que estava sendo lida em voz alta por ele.

Antes de ler cada sentença, o experimentador instruía a criança para olhar cuidadosamente os quatro desenhos e escolher apenas um único desenho - aquele que demonstrasse exatamente o que o examinador estava dizendo.

A ordem de apresentação dos tipos de sentenças foi randomizada. Também os tipos de sentenças semelhantes não foram apresentados consecutivamente.

\section{Resultados}

A Tabela 1 mostra a média de respostas corretas em função dos quantificadores, das idades e dos níveis sócioeconômicos.

Pode-se notar que o quantificador "nenhum" obteve o maior número de acertos $(\mathrm{X}=0,86)$, seguido por "todos" $(\mathrm{X}=0,51)$, "alguns" $(\mathrm{X}=0,43)$ e por último "a maioria" $(\mathrm{X}=0,33)$.

A tabela também denota que o nível de acerto foi similar entre as crianças de 5,6 e 7 anos $(X=0,55 ; 0,63 ; 0,55$ respectivamente), ficando as de 4 anos com a menor média de acertos $(X=0,40)$. Nota-se ainda que as crianças de nível sócio-econômico médio obtiveram um melhor desempenho $(\mathrm{X}=0,62)$ do que as de nível sócio-econômico baixo $(\mathrm{X}=0,45)$.
Para verificar essas conclusões, foi realizada uma Análise de Variância para medidas repetidas, tendo como fatores Idade (4) xNível Sócio-econômico (2) e Tipos de Quantificadores (4), e como Variável Dependente o número de acertos em cada tipo de quantificador.

A análise mostrou um efeito significativo para Idade $\left(\mathrm{F}_{(3,72)}=2,87, p<0.04\right)$, Nível Sócio-econômico $\left(\mathrm{F}_{(1,72)}=8,50\right.$, $p<0.005)$ e Tipos de Quantificadores $\left(\mathrm{F}_{(3,216)}=25,04\right.$, $p<0.001)$.

A média de acertos das idades foi tratada com o teste de Newman-Keuls. Esta análise revelou que as crianças de 5, 6 e 7 anos obtiveram médias de acertos significativamente superiores $(p<0,01)$ à média alcançada pelas crianças de 4 anos de idade.

Este mesmo teste aplicado às médias alcançadas nos tipos de quantificadores mostrou que o quantificador "nenhum" obteve significativamente maior número de acertos do que os quantificadores a "maioria", "alguns" $(p<0,01) \mathrm{e}$ "todos" $(p<0,05)$. Este último obteve significativamente maior número de acertos $(p<0,05)$ do que os outros dois ("a maioria" e "alguns"). Todas as outras comparações não mostraram diferenças significativas.

\section{Discussão}

Dias e Brooks (1997) enfatizam que uma das primeiras questões para desenvolver um modelo de lógica predicativa natural é caracterizar o conjunto de inferências que são realizadas rotineiramente por crianças e adultos quando compreendem sentenças envolvendo quantificação universal.

Perguntamos se os quantificadores estudados aqui seriam adquiridos pelas crianças em uma mesma idade. Os re-

Tabela 1.

Média de acertos em função dos quantificadores, idade e nível socioeconômico

\begin{tabular}{|c|c|c|c|c|c|c|c|c|c|}
\hline \multirow{2}{*}{$\begin{array}{c}\text { Idade } \\
\text { Quantificadores }\end{array}$} & \multicolumn{2}{|l|}{4} & \multicolumn{2}{|l|}{5} & \multicolumn{2}{|l|}{6} & \multicolumn{2}{|l|}{7} & \multirow{2}{*}{$\frac{\text { Média }}{\text { Total }}$} \\
\hline & NSEM & NSEB & NSEM & NSEB & NSEM & NSEB & NSEM & NSEB & \\
\hline \multirow{2}{*}{ Todos } & 0,50 & 0,30 & 0,80 & 0,20 & 0,90 & 0,20 & 0,70 & 0,50 & 0,51 \\
\hline & $(0,53)$ & $(0,48)$ & $(0,42)$ & $(0,42)$ & $(0,32)$ & $(0,42)$ & $(0,48)$ & $(0,53)$ & $(0,50)$ \\
\hline \multirow{2}{*}{ Maioria } & 0,20 & 0,20 & 0,40 & 0,30 & 0,30 & 0,50 & 0,40 & 0,30 & 0,33 \\
\hline & $(0,42)$ & $(0,42)$ & $(0,52)$ & $(0,48)$ & $(0,48)$ & $(0,53)$ & $(0,52)$ & $(0,48)$ & $(0,47)$ \\
\hline \multirow{2}{*}{ Alguns } & 0,40 & 0,40 & 0,30 & 0,50 & 0,60 & 0,60 & 0,40 & 0,20 & 0,43 \\
\hline & $(0,52)$ & $(0,52)$ & $(0,48)$ & $(0,53)$ & $(0,52)$ & $(0,52)$ & $(0,52)$ & $(0,42)$ & $(0,50)$ \\
\hline \multirow{2}{*}{ Nenhum } & 0,90 & 0,30 & 1,00 & 0,90 & 1,00 & 0,90 & 1,00 & 0,90 & 0,86 \\
\hline & $(0,32)$ & $(0,48)$ & $(0,00)$ & $(0,32)$ & $(0,00)$ & $(0,32)$ & $(0,00)$ & $(0,32)$ & $(0,35)$ \\
\hline Média total & 0,50 & 0,30 & 0,63 & 0,48 & 0,70 & 0,55 & 0,63 & 0,48 & \\
\hline
\end{tabular}


sultados encontrados mostram que não. De fato, parece existir uma ordem de aquisição e esta é dependente do nível sócio-econômico que as crianças freqüentam. O quantificador “nenhum”, entre crianças de nível sócio-econômico médio, é adquirido logo aos 4 anos de idade. As crianças de nível sócio-econômico baixo irão denotar sua compreensão um ano mais tarde. O quantificador "todos" começa a ser compreendido entre as crianças de nível sócio-econômico médio, também aos 4 anos, o que vem a corroborar os resultados de Dias e Brooks (1997) que, em seus estudos, tiveram como participantes apenas crianças deste nível social. Já entre as crianças de nível sócio-econômico baixo a compreensão do quantificador "todos" só irá acontecer por volta dos 7 anos de idade.

Como argumentam Lins e Silva e Spinillo (1998),

a criança de baixa renda usualmente inicia a escolaridade aos 7 anos, freqüentando a $1^{\underline{a}}$ série (série onde ocorre a alfabetização) que corresponde à sala de alfabetização na escola particular (crianças de 6 anos); enquanto a $1^{\underline{a}}$ série das escolas particulares corresponde à $2^{\mathrm{a}}$ série da escola pública (um ano após a alfabetização). Portanto, as séries não são equivalentes nem quanto ao conteúdo de que tratam, nem quanto aos anos escolares freqüentados pela criança ao ingressar no ensino fundamental. (p. 5).

Levanta-se então a questão se a escolarização influencia a compreensão de alguns quantificadores.

Os outros dois quantificadores têm um nível de acertos irregular independente do nível sócio-econômico e da idade. Quanto ao quantificador "alguns" aparece uma certa compreensão por parte das crianças aos 6 anos que não é detectada aos 7 anos.

Pode-se agora conjecturar que, com o aumento tanto da idade quanto da escolaridade, esses quantificadores começam a ser compreendidos de forma adequada. Uma replicação do estudo levando em conta os anos de escolarização das crianças dos dois níveis sócio-econômicos poderá esclarecer esses resultados.

Uma outra questão que tentamos investigar, a rotina direta preconizada pela Teoria da Lógica Mental e que seus defensores predizem ser aplicada corretamente mesmo por crianças pré-escolares, aqui foi observada entre crianças de nível sócio-econômico médio nos quantificadores "nenhum" e "todos". Assim, como se supunha, o modelo da lógica mental predicativa estende a teoria da lógica mental proposicional, pelo menos para este grupo.

Como sugerido por Dias e Brooks (1997) "dado o papel central da quantificação no raciocínio dedutivo, uma teoria completa de raciocínio deve incluir um componente da lógica predicativa" (p.110). As crianças, desde cedo, já começam elaborando um sistema inteiro de raciocínio dedutivo e não apenas ganhando competência na associação de pistas lingüísticas com interpretações dos quantificadores. No entanto, como argumentam essas autoras, são necessários novos estudos a fim de investigar como as interpretações de quantificadores alimentam os esquemas de inferências da lógica predicativa.

\section{Agradecimentos}

Apoio do CNPq e da FACEPE.

\section{Referências}

Bloom, L., Lahey, M., Hood, L., Lifter, K., \& Feiss, K. (1980). Complex sentences: Acquisition of syntactic connectives and the semantic relations they encode. Journal of Child Language, 7, 235-261.

Bowerman, M. (1986). First steps in acquiring conditionals. In E. Traugott, A. ter Meulen, J. S. Reilly, \& C. A. Ferguson (Orgs.), On conditionals (pp. 285-307). Cambridge: Cambridge University Press.

Braine, M. D. S., \& O'Brien, D. P. (1991). A theory of if: A lexical entry, reasoning program, and pragmatic principles. Psychological Review, 98, 182-203.

Braine, M. D. S., \& O’Brien, D. P. (1998). Mental Logic. Mahwah: Laurence Erbaum Associates.

Braine, M. D. S., \& Rumain, B. (1981). Development of comprehension of "or": Evidence for a sequence of competencies. Journal of Experimental Child Psychology, 31, 46-70.

Braine, M. D. S. (1978). On the relation between the natural logic of reasoning and standard logic. Psychological Review, 85, 1-21.

Braine, M. D. S., O'Brien, D. P., Noveck, I. A., Samuels, M., Lea, R. B., Fisch, S. M., \& Yang, Y. (1995). Predicting intermediate and multiple conclusions in propositional logic inference problems: Further evidence for a mental logic. Journal of Experimental Psychology: General, 124, 263-292.

Brainerd, C. J. (1977). On the validity of propositional logic as a model for adolescent intelligence. Interchange, 7, 40-45.

Brooks, P. J., Braine, M. D. S., Jia, X., \& Dias, M. G. (2001). Early representations for all, each, and their counterparts in Mandarim Chinese and Portuguese. In M. Bowerman \& S. C. Levinson (Orgs.), Language acquisition and conceptual development. (Cap. 11, pp. 316339). Cambridge: Cambridge University Press.

Brooks, P. J., Jia, X., Braine, M. D .S., \& Dias, M. G. (1998). A crosslinguistic study of children's comprehension of universal quantifiers: A comparison of Mandarim Chinese, Portuguese and English. First Language, 18, 33-79.

Dias, M. G. (1996). O desenvolvimento do raciocínio dedutivo. In M. G. Dias \& A. G. Spinillo (Orgs.), Tópicos em Psicologia Cognitiva (cap. 1, pp. 11-44). Recife: Editora da UFPE.

Dias, M. G., \& Brooks, P. (1997). A compreensão das crianças dos quantificadores universais. Psicologia: Teoria e Pesquisa, 13, 103-111.

Dias, M. G., \& Harris, P. L. (1988). The effect of make-believe play on deductive reasoning. British Journal of Developmental Psychology, 6, 207-221.

Dias, M. G., \& Harris, P. L. (1990). The influence of the imagination on reasoning by young children. British Journal of Developmental Psychology, \& 305-318.

Dias, M. G., \& Ruiz, E. L. (1990). Bloqueando a conversão inválida, Arquivos Brasileiros de Psicologia, 42(3), 66-77.

Ennis, R. H. (1971). Conditional logic and primary school children: A developmental study. Interchange, 2 , 126-132.

Ennis, R. H. (1975). Children's ability to handle Piaget's propositional logic. Review of Educational Research, 45, 1-41. 
Evans, J. St. B. T. (1982). The psychology of deductive reasoning. London: Routledge \& Kegan Paul.

Fein, G. G. (1981). Pretend play in childhood: An integrative review. Child Development, 52, 1095-1118.

Gelman, R., \& Meck, E. (1983). Preschoolers' counting: Principles before skill. Cognition, 13, 343-359.

Ioup, G. (1975). Some universals for quantifier scope. Syntax and Semantics, 4, 37-58.

Knifong, J. D. (1974). Logical abilities of young children - two styles of approach. Child Development, 45, 78-83.

Kuhn, D. (1977). Conditional reasoning in children. Developmental Psychology, 13, 342-353.

Lea, R. B. (1998). Logical Inferences and comprehension: How Mental Logic and text processing theories need each other. In M. D. S. Braine \& D. P. O’Brien (Orgs.), Mental Logic (cap. 5, pp. 63-78). Mahwah: Laurence Erbaum Associates.

Lea, R. B., O’Brien, D. P., Fisch, S. M., Noveck, I. A., \& Braine, M. D. S. (1990). Predicting propositional logic inferences in text comprehension. Journal of Memory and Language, 29, 361-387.

Leslie, A. M. (1987). Pretense and representation: The origins of "theory of mind." Psychological Review, 94, 412-426.

Lins e Silva, M. E. \& Spinillo, A. G. (1998). Uma análise comparativa da escrita de histórias por alunos de escolas públicas e particulares. Revista Brasileira de Estudos Pedagógicos, 79, 193, 5-16

Markovits, H. (1984). Awareness of the "possible" as a mediator of formal thinking in conditional reasoning problems. British Journal of Psychology, 75, 367-376.

Markovits, H. (1985). Incorrect conditional reasoning among adults: Competence or performance? British Journal of Psychology, 76, 241-247.

Markovits, H. (1993). The development of conditional reasoning: A Piagetian reformulation of mental models theory. Merrill-Palmer Quarterly, 39, 131-158.

Matalon, B. (1962). Étude génétique de l'implication [A genetic study of implication]. Études d'epistemologie génétique. Vol. 16. Implication, formalisation et loqique naturelle, 69-93.

Moshman, D. (1979). Development of formal hypothesis-testing ability. Developmental Psychology, 15, 104-112.
O'Brien, D. P. (1993). Mental logic and irrationality: We can put a man on the moon, so why can't we solve those logical reasoning tasks? In $\mathrm{K}$. I. Manktelow \& D. E. Over (Orgs.), Rationality: Psychological and philosophical perspectives (pp. 110-135). London: Routledge.

O’Brien, D. P. (1995). Finding logic in human reasoning requires looking in the right places. In S. E. Newstead \& J. St. B. T. Evans (Orgs.), Perspectives on thinking and reasoning: Essays in honour of Peter Wason (pp. 189-216). Hove: Lawrence Erlbaum Associates.

O’Brien, D. P., Braine, M. D. S., Connell, J. W., Noveck, I. A., Fisch, S. M., \& Fun, E. (1989). Reasoning about conditional sentences: Development of understanding of cues to quantification. Journal of Experimental Child Psychology, 48, 90-113.

O’Brien, D. P., Dias, M. G., Roazzi, A., \& Braine, M.D. (1998). Conditional reasoning: The logic of supposition and children's understanding of pretence. In M. D. S. Braine \& D. P. O'Brien (Orgs.). Mental Logic (cap.10, pp. 245-272), Mahwah: Laurence Erbaum Associates.

Overton, W. F. (1990). Competence and procedures: Constraints on the development of logical reasoning. In W. F. Overton (Org.), Reasoning, necessity, and logic: Developmental perspectives (pp. 1-32). Hillsdale: Lawrence Erlbaum Associates.

Paris, S. (1973). Comprehension of language connectives and propositional logic relationships. Journal of Experimental Child Psychology, 16, 278-291.

Reilly, J. S. (1986). The acquisition of temporals and conditionals. In E. Traugott, A. ter Meulen, J. S. Reilly \& C. A. Ferguson (Orgs.), On conditionals (pp. 309-332). Cambridge: Cambridge University Press.

Rumain, B., Connell, J. W., \& Braine, M. D. S. (1983). Conversational comprehension processes are responsible for reasoning fallacies in children as well as adults: If is not the biconditional. Developmental Psychology, 19, 471-481.

Staudenmayer, H., \& Bourne, L. (1977). Learning to interpret conditional sentences: A developmental study. Developmental Psychology, 13, 616-623.

Taplin, J. E., Staudenmayer, H., \& Taddonio, J. L. (1974). Developmental changes in conditional reasoning: Linguistic of logical? Journal of Experimental Child Psychology, 17, 360-373.

Ward, S. L., \& Overton, W. F. (1990). Semantic familiarity, relevance, and the development of deductive reasoning. Developmental Psychology, 26, 488-493.

\section{Notas}

1 Esquema para prova condicional "uma sentença como se é verdadeira quando seu antecedente juntamente com outros fatos que o falante sabe, leva ao conseqüente.” (O’Brien, Dias, Roazzi \& Braine, 1998, p. 250.)

Maria da Graça Bompastor Borges Dias, doutora em Psicologia do Desenvolvimento pela Universidade de Oxford (Reino Unido), é professora do Departamento de Psicologia da Universidade Federal de Pernambuco. Endereço para correspondência: Av. Beira Mar, 520 ap. 81, Piedade, 54310-064, Jaboatão, PE; Fone (0**81) 3271-8272; Fax (0**81) 3271-1843; e-mail: mdias@ npd.ufpe.br. Avany Bernardino Corrêa Sobral é estudante do Programa de Pós-graduação em Psicologia da UFPE. Lisandra Mendonça de Carvalho é estudante do Curso de Graduação em Psicologia da UFPE e Bolsista de IC do CNPq. 
Apêndice

FIGURAS UTILIZADAS NO TREINAMENTO
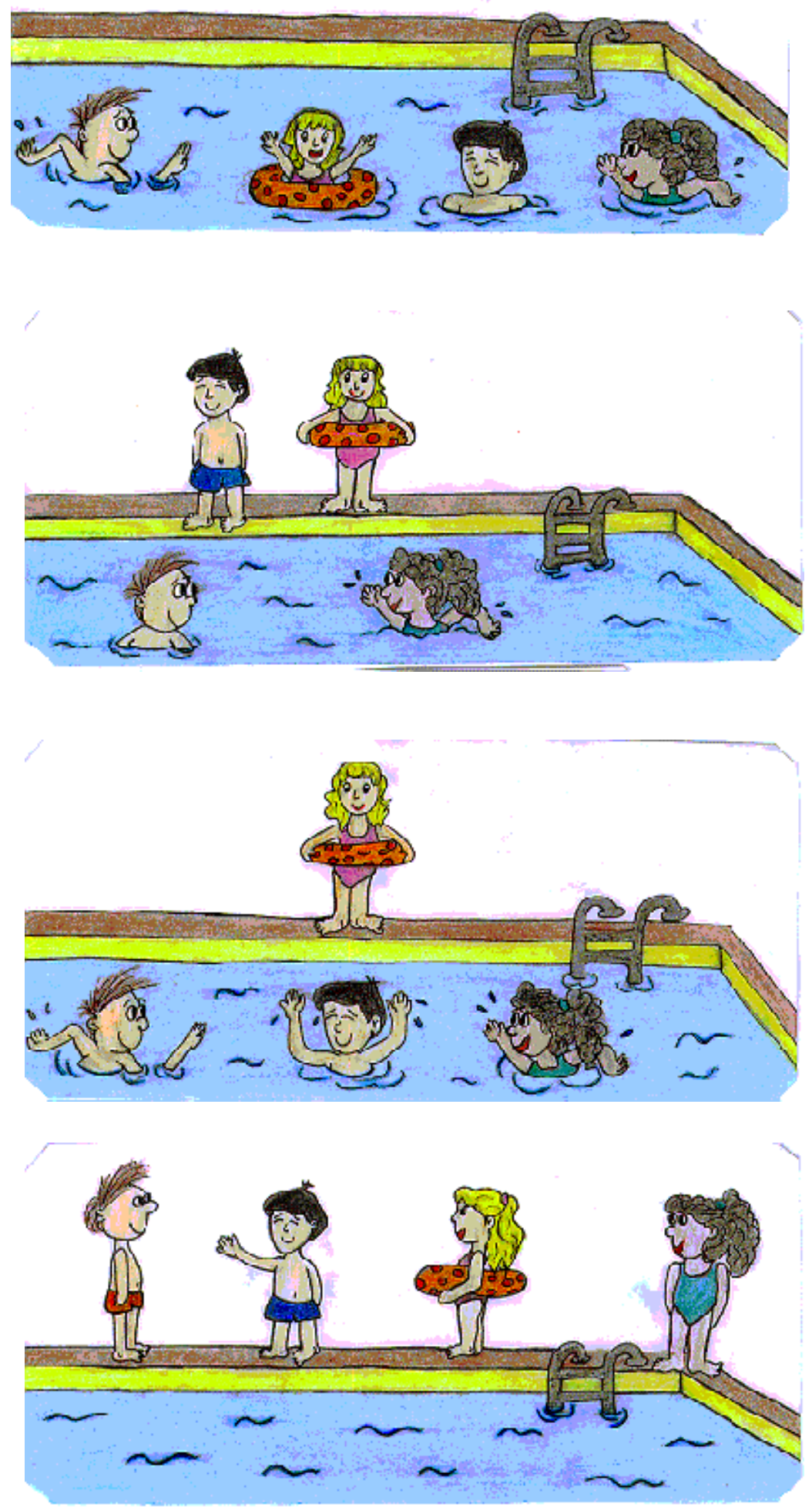